\title{
Low-Dimensional Description of Pulses under the Action of Global Feedback Control
}

\author{
Y. Kanevsky*, A.A. Nepomnyashchy \\ Department of Mathematics, Technion - Israel Institute of Technology Haifa 32000, Israel
}

\begin{abstract}
The influence of a global delayed feedback control which acts on a system governed by a subcritical complex Ginzburg-Landau equation is considered. The method based on a variational principle is applied for the derivation of low-dimensional evolution models. In the framework of those models, one-pulse and two-pulse solutions are found, and their linear stability analysis is carried out. The application of the finite-dimensional model allows to reveal the existence of chaotic oscillatory regimes and regimes with double-period and quadruple-period oscillations. The diagram of regimes resembles those found in the damped-driven nonlinear Schrödinger equation. The obtained results are compared with the results of direct numerical simulations of the original problem.
\end{abstract}

Keywords and phrases: Ginzburg-Landau equation, delayed feedback control, finitedimensional models, solitary waves

Mathematics Subject Classification: 35B36, 93B52

\section{Introduction}

A complex Ginzburg-Landau equation (CGLE)

$$
A_{t}=A+(1+i b) A_{x x}-(s+i c)|A|^{2} A
$$

describes the behavior of an extended nonlinear system above the Hopf bifurcation point. Various types of solutions of that equation have been obtained in the case of a supercritical Hopf bifurcation $(s=1)$ [1]. However, a subcritical bifurcation $(s=-1)$ appears in many applications, e.g., in convection in binary fluids [23], in lasers [15,20], and in directional solidification [9]. In the case of subcritical Hopf bifurcation, an interplay between dispersion and nonlinear frequency shift can arrest the growth of disturbances and lead to the formation of bounded wavy patterns under some conditions [19,22]. Otherwise, the solution blows up in a finite time.

The blow-up of solutions can be prevented by means of a feedback control $[10,16]$ that stabilizes localized pulses $[12,16,21]$.

The simple shape of pulses makes it attractive to use low-dimensional models for the description of their dynamics. There exist several methods of a reduction of an original equation to a finite set of ordinary

\footnotetext{
${ }^{*}$ Corresponding author. E-mail: yuliyakanevsky@gmail.com
} 
differential equations (ODE). One of these methods utilizes the integrals of energy, momentum and a finite number of higher-order generalized moments, and it has been applied for the analysis of the dynamics of localized waves of a cubic-quintic complex Ginzburg-Landau equation (CGLE) [25]. Another method is based on a modified variational technique. Low-dimensional (ODE) models, derived by means of a variational approach, have been widely used for solving problems of nonlinear optics governed primarily by partial differential equations [14], including non-conservative problems [8,24]. In the case of the complex Ginzburg-Landau equation both methods lead to identical or similar models [12]. Recently, it has been demonstrated that low-dimensional models can reproduce details of the temporal dynamics of pulses under the action of an instantaneous feedback control in an oscillatory system governed by a subcritical complex Ginzburg-Landau equation [12].

In systems with feedback control, there is usually a delay between the measurement of the system parameters by sensors and the application of control action by actuators. The influence of a delayed feedback control on the stability of bounded solutions of a subcritical Ginzburg-Landau equation has been studied in $[10,21]$.

In [13] we have applied the variational approach to modeling a delayed feedback control acting on a system governed by a subcritical Ginzburg-Landau equation with real coefficients.

In the present paper, the subcritical complex Ginzburg-Landau equation with a delayed feedback control term is used as the object for the application of the variational approach mentioned above. In Sec. 2 we discuss the variational approach and derive a finite-dimensional dynamical model which is used for the description of the behavior of the system. The linear stability of pulse solutions is analyzed in Sec. 3 In Sec. 4 the dynamics of the system in the framework of the derived ODE-models is investigated. The results of a direct numerical simulation of the original equation are discussed in Sec. 5 Sec. 6 contains concluding remarks.

\section{Variational approach}

The analysis of dynamical regimes of infinite-dimensional nonlinear systems can be simplified by reduction to finite-dimensional models based on an approximate variational approach. Here we give a brief scheme of this method. The evolution equation governing a dissipative system, which is described by a complex function $A(x, t)$, is written in the following form:

$$
F[A]=Q,
$$

where $F[A]$ is a conservative part of the equation, which corresponds to a Lagrangian density $L\left[A, A^{*}\right]$, i.e.,

$$
\frac{d}{d t} \frac{\partial L}{\partial A_{t}^{*}}+\frac{d}{d x} \frac{\partial L}{\partial A_{x}^{*}}-\frac{\partial L}{\partial A^{*}}=F[A]
$$

and $Q$ is a nonconservative part of the equation. With an ansatz of the form

$$
A=A\left(b_{1}(t), b_{2}(t), \ldots, b_{N}(t), x\right),
$$

where $b_{1}(t), b_{2}(t), \ldots, b_{N}(t)$ are parameters characterizing the shape of the solution, the variational technique [8] leads to the following system of equations

$$
\begin{gathered}
\frac{d}{d t} \frac{\partial \mathcal{L}}{\partial\left(b_{j}\right)_{t}}-\frac{\partial \mathcal{L}}{\partial b_{j}}=2 \operatorname{Re} \int_{-\infty}^{\infty} Q \frac{\partial A^{*}}{\partial b_{j}} d x, \\
j=1, \ldots, N
\end{gathered}
$$

where

$$
\mathcal{L}=\int_{-\infty}^{\infty} L\left[A\left(b_{1}(t), b_{2}(t), \ldots, b_{N}(t), x\right), A^{*}\left(b_{1}(t), b_{2}(t), \ldots, b_{N}(t), x\right)\right] d x
$$


The derivation is given in the Appendix.

We consider a system near the instability threshold governed by the subcritical complex GinzburgLandau equation under a feedback control with delay

$$
A_{t}=A+(1+i b) A_{x x}-(-1+i c)|A|^{2} A+K(A) A,
$$

where the subscripts denote partial derivatives, $K(A)$ is a control functional with a delay (see [16, 21]):

$$
K(A)=-p \max _{x}|A(x, t-\tau)| .
$$

This way of control can be achieved by changing the global parameters of the system that influence the linear growth rate of the primary instability. For instance, in the case of a morphological instability of the solidification front it is implemented by changing the sample velocity and applied temperature gradient [16], in the case of a Marangoni instability it is sufficient to change the applied heat flux, etc. In order to avoid the blow-up that can develop in any spatial point, it is natural to measure and to control the maximum value of a characteristic variable (i.e., front deformation in the case of a morphological instability) over the whole region. Near the instability threshold, the deviation of that variable from its undisturbed value will be proportional to $\max _{x}|A|$. The simplest way of control, that we consider here, is making the deviation of the growth rate proportional to that quantity with the coefficient $p$. Parameter $\tau$ corresponds to the time delay between the measurements and the control action.

Equation (2.6) has the following exact pulse solutions [11,17-19]:

$$
A(x, t)=\frac{C}{\cosh \kappa x} \exp \{i[\gamma \ln \cosh \kappa x-\Omega t]\},
$$

where $C, \kappa, \gamma$ and $\Omega$ can be computed for all values of $b$ and $c$. In the case of a feedback control of the form (2.7) it holds that

$$
\begin{aligned}
C_{ \pm} & =\frac{p}{2(1-\alpha)}\left[1 \pm \sqrt{1-4(1-\alpha) / p^{2}}\right]>0, \\
\alpha & =\frac{1}{12\left(1+b^{2}\right)}\left[\sqrt{9(b c-1)^{2}+8(b+c)^{2}}-3(b c-1)+4 b(b+c)\right], \\
\gamma & =\frac{1}{b+c}\left[6 \alpha\left(1+b^{2}\right)+3(b c-1)-2 b(b+c)\right], \\
\kappa_{ \pm}^{2} & =C_{ \pm}^{2} \frac{1}{3 \gamma} \frac{b+c}{1+b^{2}}, \\
\Omega_{ \pm} & =b \kappa_{ \pm}^{2}-\gamma \kappa_{ \pm}^{2}+c C_{ \pm}^{2} .
\end{aligned}
$$

Note that the appearance of two branches of pulse solutions was observed formerly in the case of a nonlinear Schrödinger equation disturbed by a parametric pumping and dissipation [2].

Equation (2.6) can be written in the form

$$
i A_{t}+b A_{x x}-c|A|^{2} A=i\left((1+K(A)) A+A_{x x}+|A|^{2} A\right) .
$$

The right-hand side of (2.10) is the nonconservative part, $Q$. The Lagrangian density of the left-hand side of (2.10) is as follows:

$$
L=\frac{i}{2}\left(A A_{t}^{*}-A^{*} A_{t}\right)+b\left|A_{x}\right|^{2}+\frac{c}{2}|A|^{4} .
$$

For studying the dynamics of a one-pulse solution, we use the ansatz compatible with the exact pulse solution (2.8) (see [12]),

$$
A(x, t)=\frac{C(t)}{\cosh [\kappa(t) x]} \exp \{i[\gamma(t) \ln \cosh [\kappa(t) x]+\phi(t)]\},
$$


with $C(t), \kappa(t), \gamma(t)$ and $\phi(t)$ playing the role of $b_{1}(t), \ldots, b_{4}(t)$. In this case the control functional can be written in the form $K(A)=-p C(t-\tau)$. The obtained evolution equations for $C(t), \kappa(t), \gamma(t)$ and $\phi(t)$ are as follows:

$$
\begin{aligned}
C_{t}(t)= & \frac{1}{9} C(t)\left[9-9 p C(t-\tau)+8 C^{2}(t)-\left(\gamma^{2}(t)+6 b \gamma(t)+7\right) \kappa^{2}(t)\right], \\
\kappa_{t}(t)= & \frac{4}{9} \kappa(t)\left[C^{2}(t)-\left(3 b \gamma(t)+2-\gamma^{2}(t)\right) \kappa^{2}(t)\right], \\
\gamma_{t}(t)= & \frac{2}{3} C^{2}(t)(c-\gamma(t))+\frac{2}{3} \kappa^{2}(t)(2 b-\gamma(t))\left(1+\gamma^{2}(t)\right), \\
\phi_{t}(t)= & -\frac{1}{9} C^{2}(t)[6 \ln 2(\gamma(t)-c)-4(\gamma(t)-3 c)] \\
& +\frac{1}{9} \kappa^{2}(t)\left[(6 \ln 2-4)(2 b-\gamma(t))\left(1+\gamma^{2}(t)\right)-7 b-b \gamma^{2}(t)+6 \gamma(t)\right] .
\end{aligned}
$$

According to definitions, $C(t)$ and $\kappa(t)$ are non-negative.

The stationary non-trivial solutions of model (2.13) are obtained by letting $C_{t}=\kappa_{t}=\gamma_{t}=0$ and $\phi_{t}=-\Omega$. The exact solution (2.9) is reproduced.

For the investigation of interaction of two distant pulse-like solutions, we use the ansatz $A=A_{1}+A_{2}$, $A_{j}=R_{j} \exp \left[i \theta_{j}\right]$, where

$$
\begin{aligned}
R_{j}(x, t) & =\frac{C_{j}(t)}{\cosh \left[\kappa_{j}(t)\left(x-x_{j}\right)\right]}, \\
\theta_{j}(x, t) & =\gamma_{j}(t) \ln \cosh \left[\kappa_{j}(t)\left(x-x_{j}\right)\right]+\phi_{j}(t), j=1,2
\end{aligned}
$$

for each of the solitary wave, which leads to the following variational model (the overlap of solitary waves is disregarded)

$$
\begin{aligned}
\dot{C}_{j}(t)= & \frac{1}{9} C_{j}(t)\left[9-9 p \max \left\{C_{1}(t-\tau), C_{2}(t-\tau)\right\}+8 C_{j}^{2}(t)\right. \\
& \left.-\left(\gamma_{j}^{2}(t)+6 b \gamma_{j}(t)+7\right) \kappa_{j}^{2}(t)\right], \\
\dot{\kappa}_{j}(t)= & \frac{4}{9} \kappa_{j}(t)\left[C_{j}^{2}(t)-\left(3 b \gamma_{j}(t)-\gamma_{j}^{2}(t)+2\right) \kappa_{j}^{2}(t)\right], \\
\dot{\gamma}_{j}(t)= & \frac{2}{3} C_{j}^{2}(t)\left[c-\gamma_{j}(t)\right]+\frac{2}{3} \kappa_{j}^{2}(t)\left[2 b-\gamma_{j}(t)\right]\left[1+\gamma_{j}^{2}(t)\right], \quad j=1,2 .
\end{aligned}
$$

Equations for $\dot{\phi}_{j}(t)$ do not influence the dynamics and are not written here.

\section{Linear stability analysis}

In the present section, we consider the stability of pulse solutions (2.8), (2.9) in the framework of the finite-dimensional dynamical models (2.13) and (2.15).

\subsection{One-pulse solution}

We linearize system (2.13) around solution (2.9). The linear growth rate of the perturbed solution, $\lambda$, is a solution of the following characteristic equation

$$
\lambda^{3}+a_{2}(\lambda) \lambda^{2}+a_{1}(\lambda) \lambda+a_{0}(\lambda)=0,
$$


where

$$
\begin{aligned}
a_{2}(\lambda)= & \frac{1}{9}\left[C\left(9 p e^{-\lambda \tau}-16 C\right)+2 \kappa^{2}\left(2 \gamma^{2}+9 b \gamma+17\right)\right], \\
a_{1}(\lambda)= & \frac{2}{81} \kappa^{2}\left[C\left(9 p e^{-\lambda \tau}-16 C\right)\left(2 \gamma^{2}+9 b \gamma+17\right)\right. \\
& \left.+4 \kappa^{2}\left(64+18 b^{2}+11 \gamma^{2}+36 b^{2} \gamma^{2}+69 b \gamma+\gamma^{4}-3 b \gamma^{3}\right)\right], \\
a_{0}(\lambda)= & \frac{16}{9} \kappa^{4}\left(1+b^{2}\right)\left(2+\gamma^{2}\right)\left(2-2 p C+p C e^{-\lambda \tau}\right) .
\end{aligned}
$$

The monotonic instability boundary is obtained by setting $\lambda=0$ in (3.1), which is equivalent to a condition $p C=2$, a merging point of two branches of the localized solution. A computer test of eq. (3.1) for a wide range of values of $b, c, p$ and $\tau$ reveals an existence of a positive eigenvalue for the upper branch of the solution. Thus, we conclude that the upper branch is unstable, and provide the further analysis for the lower branch of the stationary solution, with $C=C_{-}$.

The oscillatory instability boundary is obtained by substituting $\lambda=i w$ in (3.1). The following system of equations is obtained:

$$
\begin{array}{r}
-w^{3}-\Im\left(a_{2}\right) w^{2}+\Re\left(a_{1}\right) w+\Im\left(a_{0}\right)=0, \\
-\Re\left(a_{2}\right) w^{2}-\Im\left(a_{1}\right) w+\Re\left(a_{0}\right)=0 .
\end{array}
$$

First, we can find asymptotes for $p C \rightarrow 2$ and for $p \rightarrow \infty$. For $p C \rightarrow 2$ it holds $C \rightarrow 1 / \sqrt{1-\alpha}$ and $w \rightarrow 0$, therefore $\sin (w \tau) \sim w \tau$ and $\cos (w \tau) \sim 1$. Then from (3.4) it follows:

$$
\begin{aligned}
\tau= & \frac{1}{72\left(1+b^{2}\right)(b+c)\left(2+\gamma^{2}\right)}\left[3 \gamma\left(1+b^{2}\right)\left(2 \gamma^{2}+9 b \gamma+17\right)(1-9 \alpha)\right. \\
& \left.+2(b+c)\left(18 b^{2}+11 \gamma^{2}+36 b^{2} \gamma^{2}+\gamma^{4}+3 b \gamma^{3}+69 b \gamma+64\right)\right] .
\end{aligned}
$$

For $b=-0.5, c=-2.0$ it follows $\tau=-0.134$. The negative sign of $\tau$ represents the fact that in the absence of delay the solution is stable only starting from some value of $p>2 \sqrt{1-\alpha}$, see Figure 1 . The sufficient stability condition for $\tau=0, p \geq 4 \sqrt{2} / 3$, has been already found in [12].

For $p \rightarrow \infty$ it holds $C \sim 1 / p$ and thus in the leading order we obtain

$$
\begin{aligned}
-w^{3}+w^{2} \sin (w \tau) & =0, \\
w \cos (w \tau) & =0 .
\end{aligned}
$$

Assuming $w \neq 0$ we obtain from (3.7) that $w \tau=\pi / 2$. Then from (3.6) it follows $w=1$ and therefore $\tau=\pi / 2$ for $p \rightarrow \infty$.

The dependence of a delay parameter $\tau$ on a control parameter $p$ can be found analytically. From (3.3) and (3.4) one can express $\sin (w \tau)$ and $\cos (w \tau)$ in terms of $w, p$ and $\kappa(p)$. Using the trigonometric identity $\sin ^{2}(u)+\cos ^{2}(u)=1$ one obtains an equation for $w$ and then derives the expression for $\tau$. The latter expression is not written here because of its complicated form.

For $b=-0.5, c=-2.0$ the oscillatory stability boundary is shown in Figure 1. Note that a similar stability diagram was obtained for the parametrically driven [2] and ac-driven [3] damped nonlinear Schrödinger equation. The end point of the oscillatory instability boundary is marked by a circle and corresponds to $p=2 \sqrt{1-\alpha(-0.5,-2)} \approx 0.8838$ and $\tau \approx-0.134$. Note that negative values of $\tau$ contradict to the causality principle, and hence they have no physical meaning.

In addition to the oscillatory instability mode caused by the delay, a one-pulse solution can be unstable with respect to the appearance of the second pulse. That kind of instability can be considered in the framework of the model (2.15). We linearize the system (2.15) around the solution (2.9) for $A_{1}$ and around a solution $(0,0, \gamma)$ for $A_{2}$. The linear growth rate of the perturbed solution, $\lambda$, is a solution of the following characteristic equation

$$
\lambda^{2}(1-p C-\lambda)\left(\lambda^{3}+a_{2}(\lambda) \lambda^{2}+a_{1}(\lambda) \lambda+a_{0}(\lambda)\right)=0,
$$




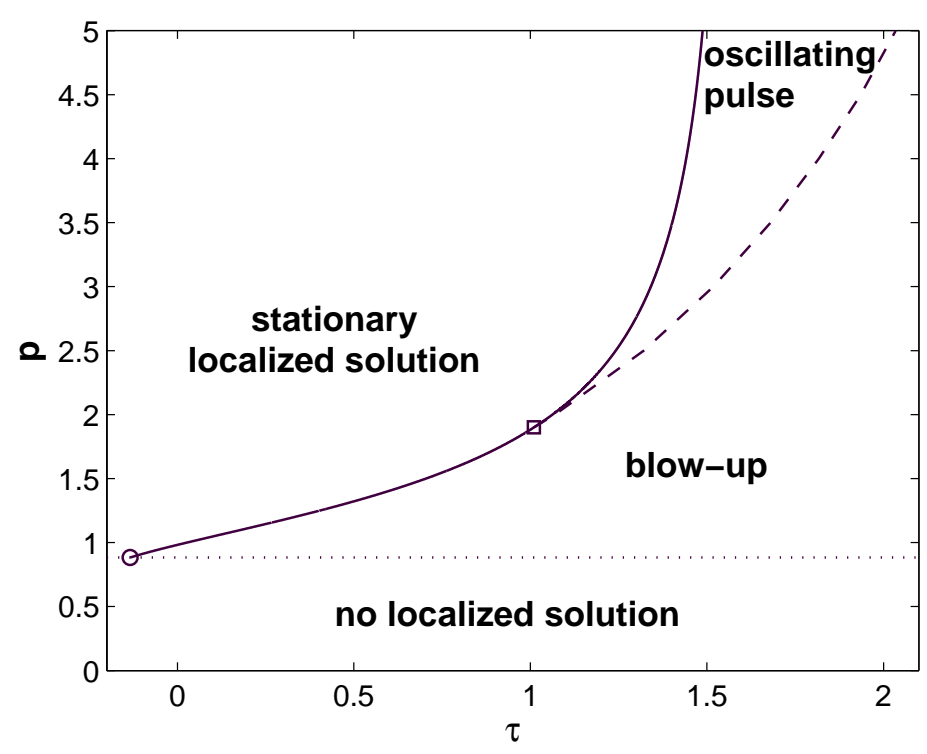

FiguRE 1. Stability boundary (solid line) for the stationary solution and the blow-up boundary (dashed line) for the oscillating solution for $b=-0.5$ and $c=-2$. The circle corresponds to $p \approx 0.8838$ and $\tau \approx-0.134$. The square at $p \approx 1.9, \tau \approx 1.01$ corresponds to the merging point of the stability and the blow-up boundaries. Dotted line: existence boundary for the stationary solution.

where $a_{2}, a_{1}, a_{0}$ are given by (3.2). The boundary $\lambda=0$ is equivalent to conditions $a_{3}=0$ or $1-p C=0$, that is, to conditions $p C=2$ or $p C=1$. The latter condition is equivalent to conditions $c=-b \pm$ $3 \sqrt{1+b^{2}}$. Therefore, for $1-p C>0$ it holds $\lambda=1-p C>0$ and the single-pulse solution is unstable. For $1-p C<0$ it holds $\lambda=1-p C<0$ and the single-pulse solution is stable for $C=C_{-}$.

\subsection{Two-pulse solution}

The monotonic stability of a two-pulse solution can be studied by linearizing the system (2.15) around the solution (2.9) for both $A_{1}$ and $A_{2}$ and assuming a certain relation between the perturbations $\tilde{C}_{1}, \tilde{C}_{2}$, e.g., $\tilde{C}_{1}>\tilde{C}_{2}$. Then the characteristic equation is as follows:

$$
\left(\lambda^{3}+a_{2}(\lambda) \lambda^{2}+a_{1}(\lambda) \lambda+a_{0}(\lambda)\right)\left(\lambda^{3}+\hat{a}_{2} \lambda^{2}+\hat{a}_{1} \lambda+\hat{a}_{0}\right)=0,
$$

where $a_{2}, a_{1}, a_{0}$ are given by (3.2) and

$$
\begin{aligned}
\hat{a}_{2}= & \frac{2}{9} \kappa^{2}\left[1-15 b \gamma+10 \gamma^{2}\right], \\
\hat{a}_{1}= & \frac{8}{81} \kappa^{4}\left[4\left(\gamma^{2}-3 b \gamma-2\right)\left(2 \gamma^{2}+9 b \gamma+17\right)+(64\right. \\
& \left.\left.+18 b^{2}+11 \gamma^{2}+36 b^{2} \gamma^{2}+69 b \gamma+\gamma^{4}-3 b \gamma^{3}\right)\right], \\
\hat{a}_{0}= & \frac{32}{9} \kappa^{4}\left(1+b^{2}\right)\left(2+\gamma^{2}\right)(1-p C) .
\end{aligned}
$$

The boundary $\lambda=0$ is equivalent to conditions $a_{0}=0$ or $\hat{a}_{0}=0$, that is, to conditions $p C=2$ or $p C=1$. The latter condition is equivalent to conditions $c=-b \pm 3 \sqrt{1+b^{2}}$. For $1-p C<0$ it holds $\hat{a}_{0}<0$ therefore at least one of the eigenvalues $\lambda$ is positive. For $1-p C>0$ it holds $\hat{a}_{0}>0$ and hence 
$\lambda<0$. Thus the two-pulse solution is unstable for $1-p C<0$ and stable for $1-p C>0$ and $C=C_{-}$, and this is opposite to the stability of the single-pulse solution, at least if we do not consider the possibility of the oscillatory instability. Note that multipulse solitons were studied formerly for the parametrically driven $[5,6]$ and ac-driven [4] damped nonlinear Schrödinger equation.

\section{Dynamics governed by ODE-model}

The investigation of interaction of two pulse-like solutions has been provided in the framework of the obtained variational model (2.15). The results are depicted in Figure 2 and Figure 3.

a)

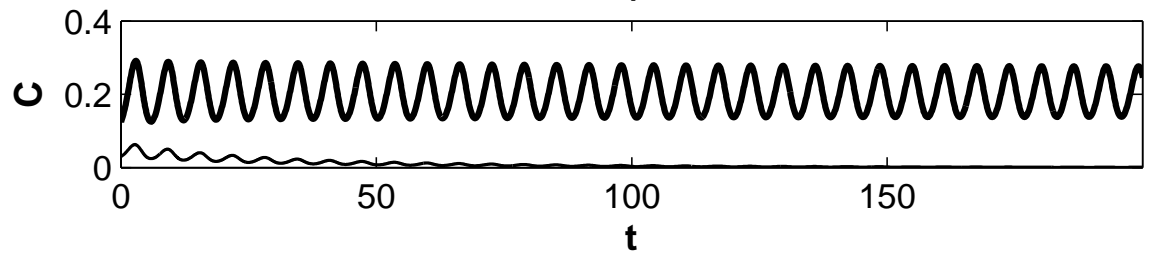

b)

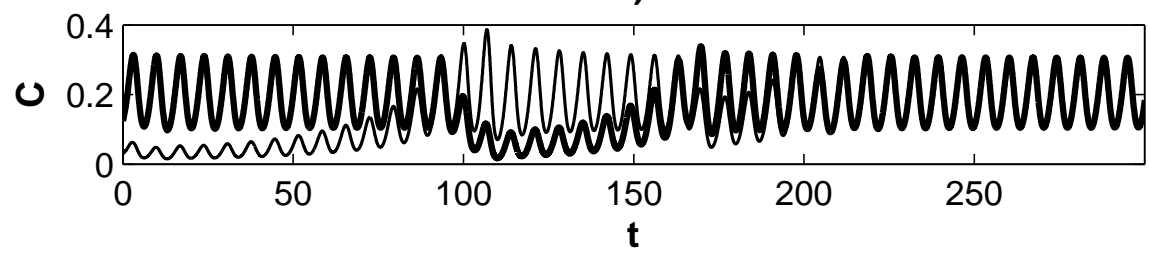

c)

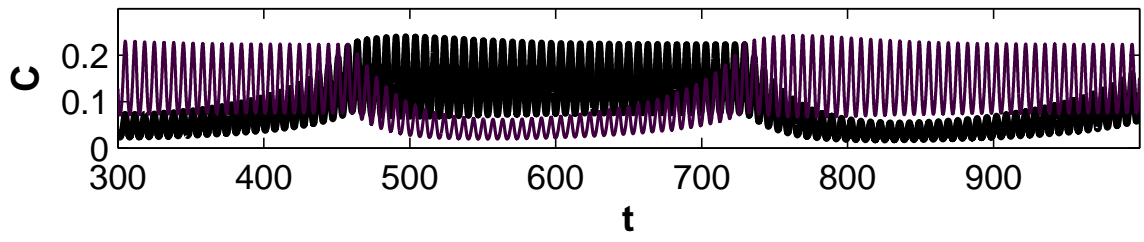

Figure 2. Dynamical regimes for a) $b=-0.5, c=2, p=5$ and $\tau=1.5$; b) $b=-1$, $c=10, p=5$ and $\tau=1.6$; c) $b=2, c=8, p=7$ and $\tau=1.6$. Thick solid line: $C_{1}(t)$, thin solid line: $C_{2}(t)$.

In the region of parameters $\left\{-b-3 \sqrt{1+b^{2}}<c<-b+3 \sqrt{1+b^{2}}\right\}$ a one-pulse oscillating solution is obtained for appropriate values of a delay parameter, see Figure 2(a). The dynamics of a one-pulse oscillating solution for the case $b=c=0$ is described in details in [13] in the framework of model (2.13).

A two-pulse solution is observed in the region $\left\{c<-b-3 \sqrt{1+b^{2}}\right\} \cup\left\{c>-b+3 \sqrt{1+b^{2}}\right\}$, where a one-pulse solution becomes unstable. The obtained regimes of two coexisting oscillating pulses [Figure 2(b)] and two competing oscillating pulses [Figure 2(c)] have been already observed in direct numerical simulations of (2.6) in [10]. However, some new dynamical regimes were obtained in the case of two coexisting pulses with the increase of the delay parameter. These regimes were analyzed with the help of the Poincare mappings. For fixed values of $p$ and $\tau$ we have measured the successive maximal values $C_{n}$ of $C_{1}(t)$, and analyzed the dependence $f\left(C_{n}\right)=C_{n+1}$. The obtained mappings are represented in Figure $3(\mathrm{~b}),(\mathrm{d}),(\mathrm{f})$. It was found out that the increasing of the delay leads to an apparently chaotic 
oscillation of two pulses, see Figure 3(c). These chaotic oscillations have 'windows' of periodic oscillating regimes, for some values of the delay parameter. The doubling and quadrupling of the period of the oscillations were observed, see Figure 3(a),(e).

a)

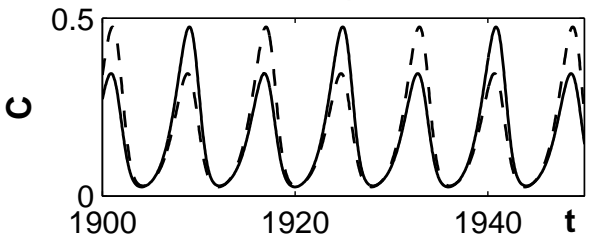

c)

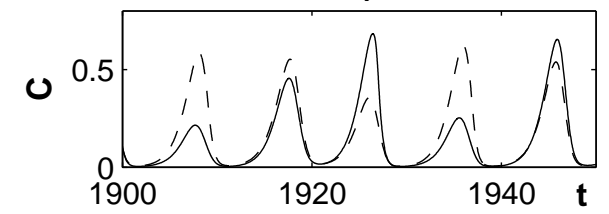

e)

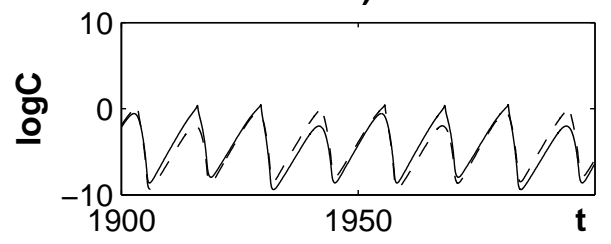

b)

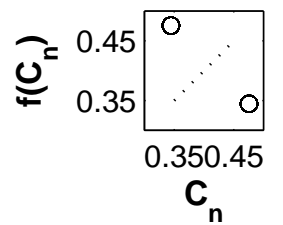

d)

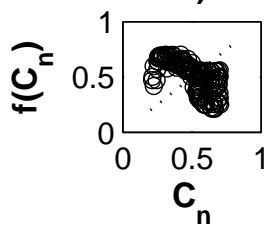

f)

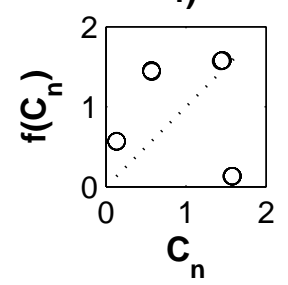

Figure 3. Dynamical regimes and corresponding Poincare mappings for $p=5, b=-1$, $c=12$ and: a),b) $\tau=1.65$; c),d) $\tau=1.8$ and e),f) $\tau=2.0$. Solid line: $C_{1}(t)$, dashed line: $C_{2}(t)$, circles: $f\left(C_{n}\right)=C_{n+1}$, dotted line: $f\left(C_{n}\right)=C_{n}$.

\section{Direct numerical simulation}

The dynamical regimes, predicted by the ODE-model and demonstrated in Figure 2, have been already observed in [10] by means of a direct numerical simulation of (2.6). Therefore, in the present paper a direct numerical simulation of (2.6) is provided in order to obtain the new dynamical regimes predicted by the finite-dimensional model for a two-pulse solution (shown in Figure 3). For this purpose, we have used a pseudospectral code with time integration in Fourier space, periodic boundary conditions, Crank-Nicholson scheme for the linear operator and Adams-Bashforth scheme for the nonlinear one.

The computations were held in $b=-1.0, c=12.0$ for $p=5.0$ and time steps $d t=10^{-3}$ and $d t=10^{-4}$. The results of numerical simulation with time step $d t=10^{-3}$ are presented in Figure 4, 5. The dynamical regimes shown in Figure 4(a),(c) and Figure 5 were also reproduced in numerical simulations with time step $d t=10^{-4}$, while as regime demonstrated in Figure 4(b) becomes similar to that from Figure 4(c) for time step $d t=10^{-4}$.

The onset of oscillation occurs for a delay value $\tau \approx 1.442$, while the theoretical value is $\tau \approx 1.563$. However, the prediction of the ODE-model for the blow-up boundary is more precise. The solution blows up when a delay parameter reaches the value $\tau \approx 2.049$ and the predicted value is $\tau \approx 2.062$.

The increase of a delay parameter leads to a regular oscillatory regime, see Figure 4(a), and then to chaotic oscillations. The numerical simulations of (2.6) with time step $d t=10^{-3}$ reveals two types 
of chaotic oscillations: (i) asynchronous oscillations of of pulses (see Figure 4(b)) that look similar to theoretically predicted regime shown in Figure 3(b); and (ii) synchronized oscillations of pulses with changing of their centers' positions, see Figure 4(c). The regime of synchronized chaotic oscillations was also obtained for time step $d t=10^{-4}$, but we have not managed to obtain the asynchronous chaotic regime for $d t=10^{-4}$.

With the further increase of a delay parameter a certain kind of the inverted period-doubling cascade is observed (cf. [7]). The dynamical regimes change from chaotic to quadruple-period oscillations, then to double-period oscillations, and finally to a regular periodic oscillatory regime. The example of doubleperiod oscillations is shown in Figure 5. The difference between regimes with doubled and quadrupled periods would be undistinguishable in the plots, although it can be seen from Table 1.

a)

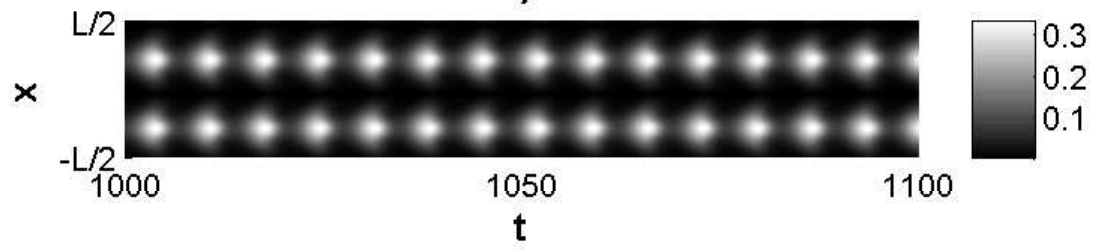

b)

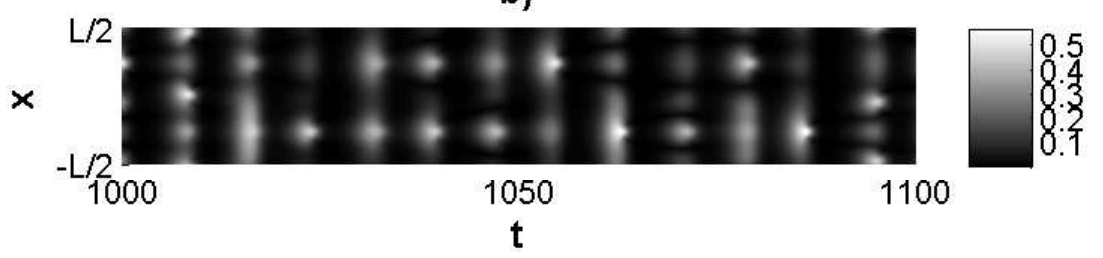

c)

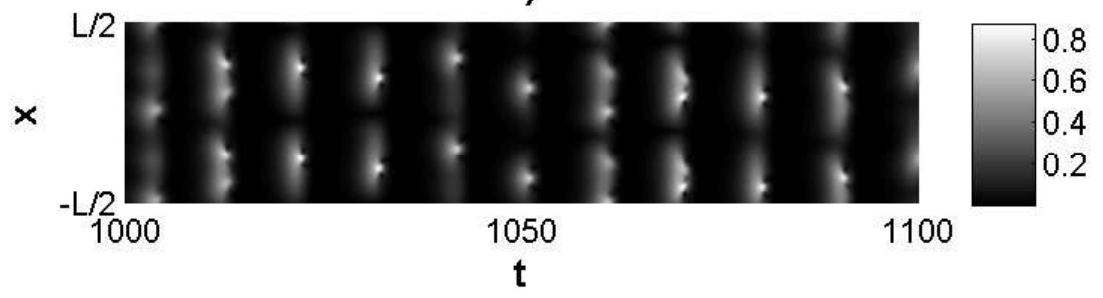

Figure 4. Spatio-temporal plots of $|A|$ for $b=-1, c=12, L=12 \pi, p=5.0$ and a) $\tau=1.5$; b) $\tau=1.6$; c) $\tau=1.75$.

\section{Conclusions}

Dynamics of subcritical oscillations under the action of a global feedback control with delay has been considered. We have constructed a finite-dimensional model for the description of the indirect interaction of pulses stabilized by the active control. The analysis presented above demonstrates that the replacement of the original delay-PDE by an approximate delay-ODE obtained by means of a modified variational approach represents a "cheap" and rather precise method for studying the stability and nonlinear dynamics of localized structures under the action of a delayed feedback control.

Using a delay-ODE model allowed to find the oscillatory stability boundary of pulses and to reveal the existence of several new dynamical regimes. Some of new regimes similar to those predicted by the 


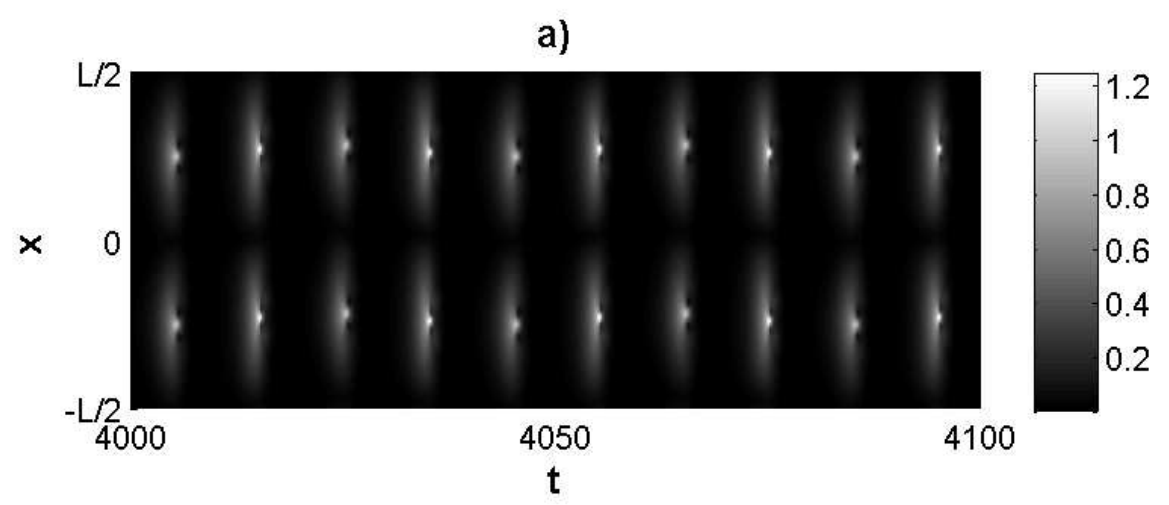

b)

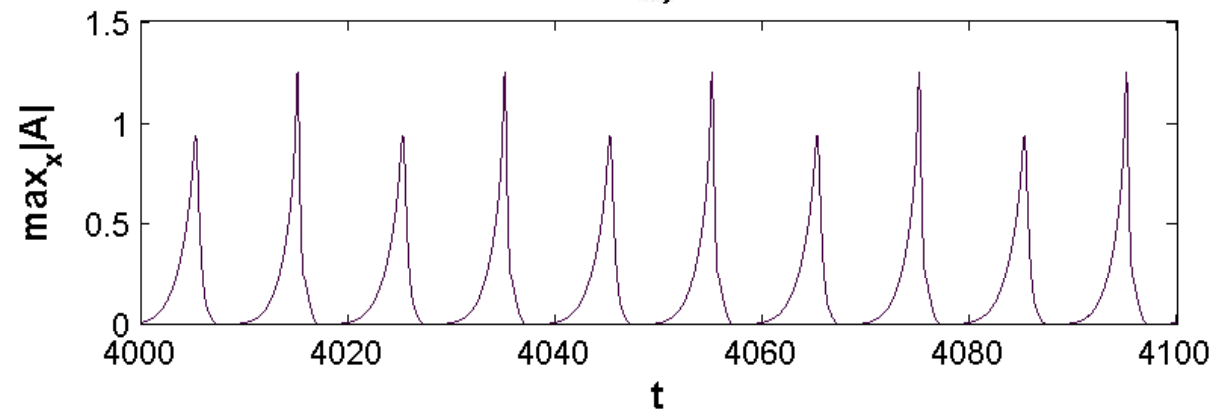

Figure 5. Dynamical regime for $b=-1, c=12, L=12 \pi, p=5.0$ and $\tau=1.865$ : a) spatio-temporal plot of $|A|$; b) plot of $\max _{x}|A|$.

TABLE 1. Dynamical regimes for different values of $\tau$ (time step $d t=10^{-4}$ ).

\begin{tabular}{cccc} 
delay value & $\tau=2.0$ & $\tau=1.864$ & $\tau=1.8635$ \\
\hline maximal & 1.2065 & 0.9324 & 0.9321 \\
values & 1.2065 & 1.2451 & 1.2495 \\
of & 1.2065 & 0.9324 & 0.9286 \\
$\max _{x}|A|$ & 1.2065 & 0.9324 & 1.2438 \\
for & 1.2065 & 1.2451 & 0.9321 \\
10 & 1.2065 & 0.9324 & 0.9286 \\
successive & 1.2065 & 1.2451 & 1.2438 \\
oscillations & 1.2065 & 0.9324 & 0.9321 \\
& 1.2065 & 1.2451 & 1.2495 \\
\hline
\end{tabular}

theoretical model were obtained by means of the direct numerical simulation if the original delay-PDE. In particular, the regime of asynchronous chaotic oscillations and oscillatory regimes with double-periods and quadruple-periods were observed. In addition, the new regime of delay-caused synchronized oscillations was obtained by the direct numerical simulation of the original equation. A similarity to regimes formerly found for the parametrically or ac-driven nonlinear Schrödinger equation has been revealed.

Acknowledgements. The support of Israel Science Foundation (grant \# 812/06) and Minerva Center for Nonlinear Physics of Complex Systems is acknowledged. This work was inspired by valuable discussions with A.A. Golovin. 


\section{Appendix}

Proof. of (2.4). We start with noting that if $u=u\left(b_{1}(t), b_{2}(t), \ldots, b_{N}(t), x\right)$, then

$$
u_{t}=\sum_{j=1}^{N} \frac{\partial u}{\partial b_{j}}\left(b_{j}\right)_{t} \quad \text { and } \quad u_{t}^{*}=\sum_{j=1}^{N} \frac{\partial u^{*}}{\partial b_{j}}\left(b_{j}\right)_{t}
$$

therefore

$$
\frac{\partial u_{t}}{\partial\left(b_{j}\right)_{t}}=\frac{\partial u}{\partial b_{j}} \quad \text { and } \quad \frac{\partial u_{t}^{*}}{\partial\left(b_{j}\right)_{t}}=\frac{\partial u^{*}}{\partial b_{j}}, \quad j=1, \ldots, N .
$$

Also, we must note that $u, u^{*}, u_{x}$ and $u_{x}^{*}$ are functions of $b_{i}(t), i=1, \ldots, N$, and $x$, therefore they are independent of $\left(b_{i}\right)_{t}$. Thus,

$$
\begin{aligned}
& \frac{d}{d t} \frac{\partial \mathcal{L}}{\partial\left(b_{i}\right)_{t}}-\frac{\partial \mathcal{L}}{\partial b_{i}}=\frac{d}{d t}\left[\int_{-\infty}^{\infty}\left(\frac{\partial L}{\partial u_{t}} \frac{\partial u_{t}}{\partial\left(b_{i}\right)_{t}}+\frac{\partial L}{\partial u_{t}^{*}} \frac{\partial u_{t}^{*}}{\partial\left(b_{i}\right)_{t}}\right) d x\right] \\
- & \int_{-\infty}^{\infty}\left[\frac{\partial L}{\partial u} \frac{\partial u}{\partial b_{i}}+\frac{\partial L}{\partial u^{*}} \frac{\partial u^{*}}{\partial b_{i}}+\frac{\partial L}{\partial u_{t}} \frac{\partial u_{t}}{\partial b_{i}}+\frac{\partial L}{\partial u_{t}^{*}} \frac{\partial u_{t}^{*}}{\partial b_{i}}+\frac{\partial L}{\partial u_{x}} \frac{\partial u_{x}}{\partial b_{i}}+\frac{\partial L}{\partial u_{x}^{*}} \frac{\partial u_{x}^{*}}{\partial b_{i}}\right] d x \\
= & \int_{-\infty}^{\infty}\left[\left(\frac{\partial}{\partial t} \frac{\partial L}{\partial u_{t}}-\frac{\partial L}{\partial u}\right) \frac{\partial u}{\partial b_{i}}+\left(\frac{\partial}{\partial t} \frac{\partial L}{\partial u_{t}^{*}}-\frac{\partial L}{\partial u^{*}}\right) \frac{\partial u^{*}}{\partial b_{i}}-\frac{\partial L}{\partial u_{x}} \frac{\partial}{\partial x} \frac{\partial u}{\partial b_{i}}-\frac{\partial L}{\partial u_{x}^{*}} \frac{\partial}{\partial x} \frac{\partial u^{*}}{\partial b_{i}}\right] d x \\
= & \int_{-\infty}^{\infty}\left[\left(Q^{*}-\frac{\partial}{\partial x} \frac{\partial L}{\partial u_{x}}\right) \frac{\partial u}{\partial b_{i}}+\left(Q-\frac{\partial}{\partial x} \frac{\partial L}{\partial u_{x}^{*}}\right) \frac{\partial u^{*}}{\partial b_{i}}-\frac{\partial L}{\partial u_{x}} \frac{\partial}{\partial x} \frac{\partial u}{\partial b_{i}}-\frac{\partial L}{\partial u_{x}^{*}} \frac{\partial}{\partial x} \frac{\partial u^{*}}{\partial b_{i}}\right] d x \\
= & 2 \operatorname{Re}\left\{\int_{-\infty}^{\infty} \frac{\partial u^{*}}{\partial b_{i}} d x\right\}-\underbrace{\int_{-\infty}^{\infty} \frac{\partial}{\partial x}\left[\frac{\partial L}{\partial u_{x}} \frac{\partial u}{\partial b_{i}}+\frac{\partial L}{\partial u_{x}^{*}} \frac{\partial u^{*}}{\partial b_{i}}\right] d x}_{=0} .
\end{aligned}
$$

\section{References}

[1] I.S. Aranson, L. Kramer. The world of the complex Ginzburg-Landau equation. Rev. Mod. Phys., 74 (2002), $99-143$.

[2] I.V. Barashenkov, M.M. Bogdan, V.I. Korobov. Stability diagram of the phase-locked solitons in the parametrically driven, damped nonlinear Schrödinger equation. Europhys. Lett., 15 (1991), 113-118.

[3] I.V. Barashenkov, Yu.S. Smirnov. Existence and stability chart for the ac-driven, damped nonlinear Schrödinger solitons. Phys. Rev. E, 54 (1996), 5707-5725.

[4] I.V. Barashenkov, Yu.S. Smirnov, N.V. Alexeeva. Bifurcation to multisoliton complexes in the ac-driven, damped nonlinear Schrödinger equation. Phys. Rev. E, 57 (1998), 2350-2364.

[5] I.V. Barashenkov, E.V. Zemlyanaya. Stable complexes of parametrically driven, damped nonlinear Schrödinger solitons. Phys. Rev. Lett., 83 (1999), 2568-2571.

[6] I.V. Barashenkov, E.V. Zemlyanaya. Soliton complexity in the damped-driven nonlinear Schrödinger equation: Stationary to periodic to quasiperiodic complexes. Phys. Rev. E, 83 (2011), 056610.

[7] M. Bondila, I.V. Barashenkov, M.M. Bogdan. Topography of attractors of the parametrically driven nonlinear Schrödinger equation. Physica D, 87 (1995), 314-320.

[8] S. Chávez Cerda, S.B. Cavalcanti, J.M. Hickmann. A variational approach of nonlinear dissipative pulse propagation. Eur. Phys. J. D, 1 (1998), 313-316.

[9] S.H. Davis. Theory of Solidification. Cambridge University Press, Cambridge, 2001.

[10] A.A. Golovin, A.A. Nepomnyashchy. Feedback control of subcritical oscillatory instabilities. Phys. Rev. E, 73 (2006), 046212 .

[11] L.M. Hocking, K. Stewartson. On the nonlinear response of a marginally unstable plane parallel flow to a twodimensional disturbance. Proc. Roy. Soc. Lond. A, 326 (1972), 289-313.

[12] Y. Kanevsky, A.A. Nepomnyashchy. Stability and nonlinear dynamics of solitary waves generated by subcritical oscillatory instability under the action of feedback control. Phys. Rev. E, 76 (2007), 066305.

[13] Y. Kanevsky, A.A. Nepomnyashchy. Dynamics of solitary waves generated by subcritical instability under the action of delayed feedback control. Physica D, 239 (2010), 87-94. 
[14] B.A. Malomed. Variational methods in nonlinear fiber optics and related fields. Progress in Optics, 43 (2002), 69-191.

[15] J.D. Moores. On the Ginzburg-Landau laxer mode-locking model with 5th order saturable absorber term. Opt. Commun., 96 (1993), 65-70.

[16] A.A. Nepomnyashchy, A.A. Golovin, V. Gubareva, V. Panfilov. Global feedback control of a long-wave morphological instability. Physica D, 199 (2004), 61-81.

[17] K. Nozaki, N. Bekki. Exact solutions of the generalized Ginzburg-Landau equation. J. Phys. Soc. Jpn., 53 (1984), 1581-1582.

[18] N.R. Pereira, L. Stenflo. Nonlinear Schrödinger equation including growth and damping. Phys. Fluids, 20 (1977), $1733-1734$

[19] S. Popp, O. Stiller, E. Kuznetsov, L. Kramer. The cubic complex Ginzburg-Landau equation for a backward bifurcation. Physica D, 114 (1998), 81-107.

[20] J.A. Powell, P.K. Jakobsen. Localized states in fluid convection and multiphoton lasers. Physica D, 64 (1993), $132-152$.

[21] B.Y. Rubinstein, A.A. Nepomnyashchy, A.A. Golovin. Stability of localized solutions in a subcritically unstable patternforming system under a global delayed control. Phys. Rev. E, 75 (2007), 046213.

[22] W. Schöpf, L. Kramer. Small-amplitude periodic and chaotic solutions of the complex Ginzburg-Landau equation for a subcritical bifurcation. Phys. Rev. Lett., 66 (1991), 2316-2319.

[23] W. Schöpf, W. Zimmermann. Convection in binary fluids - amplitude equations, codimension-2 bifurcation, and thermal fluctuations. Phys. Rev. E, 47 (1993), 1739-1764.

[24] V. Skarka, N.B. Aleksić. Stability criterion for dissipative soliton solutions of the one-, two-, and three-dimensional complex cubic-quintic Ginzburg-Landau equations. Phys. Rev. Lett., 96 (2006), 013903.

[25] E.N. Tsoy, A. Ankiewicz, N. Akhmediev. Dynamical models for dissipative localized waves of the complex GinzburgLandau equation. Phys. Rev. E, 73 (2006), 036621. 